\title{
Relaxation Mechanisms and Strain-Controlled Oxygen Vacancies in Epitaxial $\mathrm{SrMnO}_{3}$ Films
}

\author{
Eric Langenberg,* Laura Maurel, Guillermo Antorrena, Pedro A. Algarabel, César Magén, \\ and José A. Pardo*
}

Cite This: ACS Omega 2021, 6, 13144-13152

Read Online

\section{ACCESS | Lill Metrics \& More | 回 Article Recommendations ｜（） Supporting Information}

ABSTRACT: $\mathrm{SrMnO}_{3}$ has a rich epitaxial strain-dependent ferroic phase diagram, in which a variety of magnetic orderings, even ferroelectricity, and thus multiferroicity, are accessible by gradually modifying the strain. Different relaxation processes, though, including the presence of strain-induced oxygen vacancies, can severely curtail the possibility of stabilizing these ferroic phases. Here, we report on a thorough investigation of the strain relaxation mechanisms in $\mathrm{SrMnO}_{3}$ films grown on several substrates imposing varying degrees of strain from slightly compressive $(-0.39 \%)$ to largely tensile $\approx+3.8 \%$. First, we determine the strain dependency of the critical thickness $\left(t_{c}\right)$ below which pseudomorphic growth is obtained. Second, the mechanisms of stress relaxation are elucidated, revealing that misfit dislocations and stacking faults accommodate the strain above $t_{c}$. Yet, even for films thicker than $t_{c}$, the atomic monolayers below $t_{c}$ are proved to remain fully coherent. Therefore, multiferroicity may also emerge even in films that appear to be partially relaxed. Last, we demonstrate that fully coherent films with

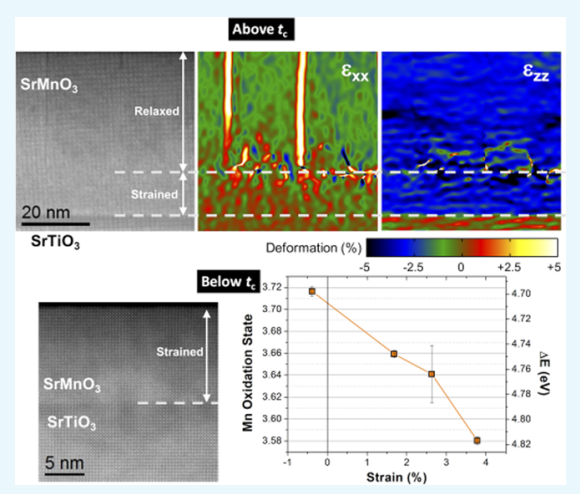
the same thickness present a lower oxygen content for increasing tensile mismatch with the substrate. This behavior proves the coupling between the formation of oxygen vacancies and epitaxial strain, in agreement with first-principles calculations, enabling the strain control of the $\mathrm{Mn}^{3+} / \mathrm{Mn}^{4+}$ ratio, which strongly affects the magnetic and electrical properties. However, the presence of oxygen vacancies $/ \mathrm{Mn}^{3+}$ cations reduces the effective epitaxial strain in the $\mathrm{SrMnO}_{3}$ films and, thus, the accessibility to the strain-induced multiferroic phase.

\section{INTRODUCTION}

Room-temperature single-phase magnetoelectric multiferroics are expected to show an efficient control of their magnetic state by means of electric fields, thus allowing a substantial decrease in the energy consumption of nonvolatile memories and other devices. ${ }^{1,2}$ In this sense, the predictions that $\mathrm{AMnO}_{3}$ (A being an alkaline-earth) could present simultaneous ferroelectricferromagnetic states under sufficiently high epitaxial strain ${ }^{3,4}$ or under sufficiently large chemical pressure (i.e., using large alkaline-earth cations like $\mathrm{Ba})^{5}$ paved the way to an intensive experimental search. As ferroelectricity in these compounds would be driven by the off-centering of the $\mathrm{Mn}^{4+}$ magnetic cation, strong magnetoelectric coupling was anticipated. ${ }^{3-5}$

Some experimental confirmations of these first-principles predictions came in recent years: incipient ferroelectricity was shown in epitaxially strained $\mathrm{CaMnO}_{3}$ films, ${ }^{6}$ ferroelectricity was measured at low temperature in $(\mathrm{Sr}, \mathrm{Ba}) \mathrm{MnO}_{3}$ crystals under Ba-induced chemical pressure, ${ }^{7}$ and the interplay between magnetic and electrical properties was shown in $(\mathrm{Sr}, \mathrm{Ba}) \mathrm{MnO}_{3}$ films with several compositions. ${ }^{8-10}$ Yet, the stability of the perovskite phase for increasingly Ba-rich $(\mathrm{Sr}, \mathrm{Ba}) \mathrm{MnO}_{3}$ compounds rapidly deteriorated, ${ }^{11}$ preventing the multiferroic character from being attained. Regarding pure epitaxial $\mathrm{SrMnO}_{3}$, the initial predictions ${ }^{4}$ were partially corroborated by optical second-harmonic generation (SHG) measurements, proving the emergence of a polar state at $300 \mathrm{~K}$ in $\mathrm{SrMnO}_{3}$ films grown under $1.7 \%$ tensile epitaxial strain ${ }^{12}$ and ferroelectricity at $4 \mathrm{~K}$ in strongly strained (3.8\%) films. ${ }^{13}$ However, all of these films remain antiferromagnetic, ${ }^{13,14}$ in agreement with previous predictions ${ }^{4}$ and with a recently calculated strain-temperature-ferroic order phase diagram. ${ }^{15}$

Epitaxial strain engineering has been widely used to tune the functional properties of ferroic thin-film oxides. ${ }^{16}$ At the same time, it has become clear that most perovskite oxides containing cations with several oxidation states can modify their stoichiometry in response to the epitaxial strain state, as it has been reviewed by Herklotz et al. ${ }^{17}$ In the case of alkalineearth manganites, a correlation between the epitaxial strain and the concentration of oxygen vacancies has been established from first-principles calculations ${ }^{18,19}$ and in X-ray absorption spectroscopy experiments performed in $\mathrm{CaMnO}_{3}$ films. ${ }^{20}$ As

Received: February 22, 2021

Accepted: March 31, 2021

Published: May 13, 2021 
(a)

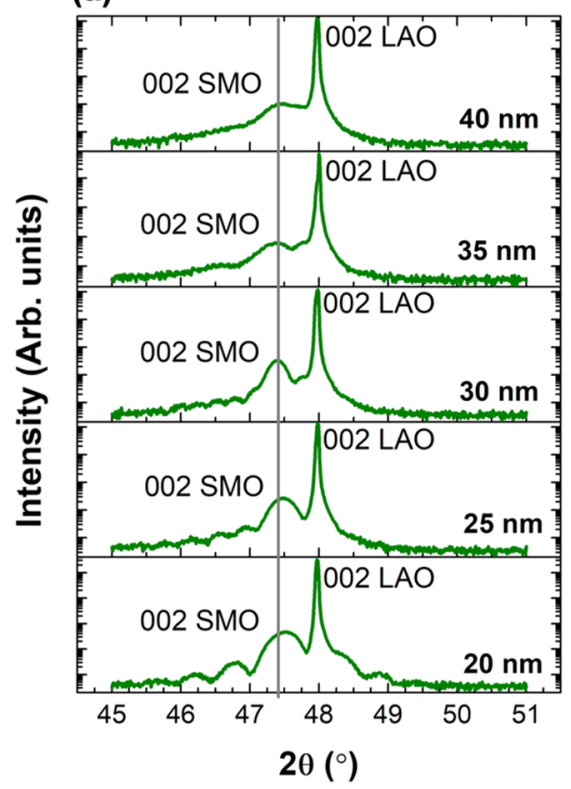

(c)

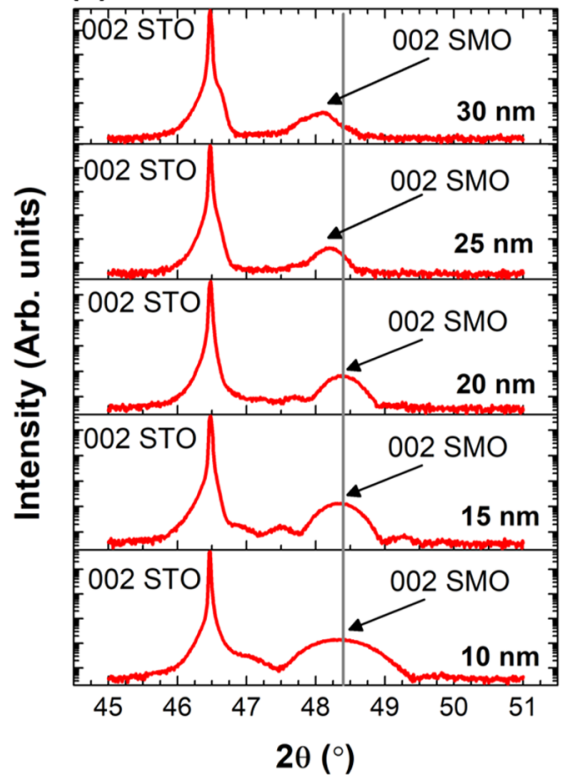

(b)

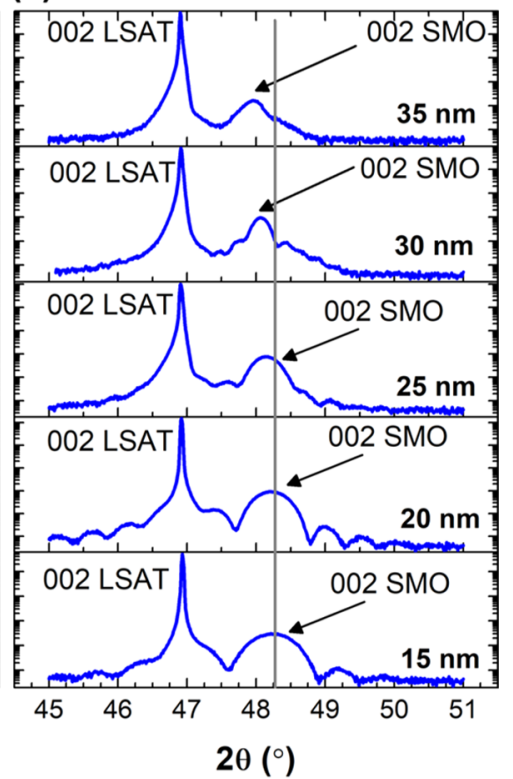

(d)

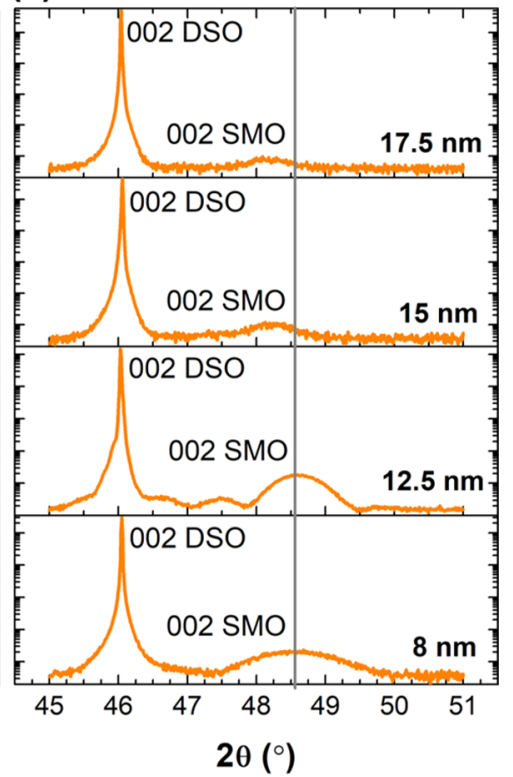

Figure 1. Thickness dependence of the $\theta / 2 \theta$ symmetrical XRD scans measured around the 002 reflection of SMO films grown on (a) LAO, (b) LSAT, (c) STO, and (d) DSO substrates.

the overall electric charge must remain neutral, the presence of oxygen vacancies is concomitant with the lowering of the average oxidation state of $\mathrm{Mn}$, giving rise to an increased cell volume relative to the stoichiometric compound due to the larger size of $\mathrm{Mn}^{3+}$ compared to $\mathrm{Mn}^{4+}$ (the ionic radii in octahedral coordination are 0.65 and $0.53 \AA$, respectively). ${ }^{21}$ Oxygen vacancies originate during the growth process as a response to the epitaxial stress imposed by the substrate, thus decreasing the effective strain of the film material. This situation gives rise to a complex scenario where epitaxial strain, oxygen stoichiometry, and functional properties are coupled. ${ }^{19}$

In the particular case of $\mathrm{SrMnO}_{3}$ (hereinafter denoted SMO), although sufficiently high epitaxial stress was predicted to induce a multiferroic state ${ }^{4,15}$ and a giant magnetoelectric cross-caloric effect, ${ }^{22}$ the same stress considerably lowers the formation energy of oxygen vacancies, which, in turn, favors ferromagnetic order to the detriment of the ferroelectric one. ${ }^{19}$ This could explain why ferroelectricity has been detected only in nearly stoichiometric strained SMO films coated with another oxide layer to prevent oxygen out-diffusion, ${ }^{13}$ while only highly oxygen-deficient films are ferromagnetic as a consequence of $\mathrm{Mn}^{3+}-\mathrm{Mn}^{4+}$ double-exchange magnetic interaction. $^{23,24}$

To control the ferroic properties of epitaxial SMO films, a clarification of the strain relaxation mechanisms is thus required. A former study ${ }^{25}$ used electron energy loss spectroscopy (EELS) to measure the oxygen vacancy concentration in ultrathin $(10 \mathrm{~nm})$ epitaxial SMO films covered with a $\mathrm{DyScO}_{3}$ capping layer aimed at preventing postdeposition oxygen exchange between the film and the environment. Here, we have prepared epitaxial SMO films on several substrates in a broad range of thickness $(5-100 \mathrm{~nm})$ to 
determine the maximum value for coherent growth. The structure and microstructure of the SMO films have been correlated with their thickness and the mismatch imposed by the substrate. All of the films were uncovered, thus allowing for oxygen diffusion during the postdeposition cooling. Lastly, we have measured the oxygen content of the thinnest, fully strained films by X-ray photoelectron spectroscopy (XPS), giving the average oxidation state of $\mathrm{Mn}$ and its dependence with the mismatch.

\section{RESULTS AND DISCUSSION}

Determination of the Critical Thickness. Generally speaking, epitaxial thin films can show pseudomorphic growth on mismatched substrates, which means that the in-plane lattice parameter of the film remains the same as that of the substrate. $^{26}$ This elastically strained growth can take place below the critical thickness $\left(t_{c}\right)$ of the film. Above this value, a partial relief of stress takes place, the in-plane lattice parameter of the film becomes different from that of the substrate (and closer to its unstrained value), and its crystal quality worsens, typically through the formation of misfit dislocations. ${ }^{26}$ The experimental determination of $t_{\mathrm{c}}$ requires the careful structural study of films in a broad range of thickness. ${ }^{27} \mathrm{We}$ systematically measured $\theta / 2 \theta$ X-ray diffraction (XRD) scans in identical conditions around the symmetric 002 reflection in a series of films with thickness between 5 and $100 \mathrm{~nm}$ deposited on $\mathrm{LaAlO}_{3},\left(\mathrm{LaAlO}_{3}\right)_{0.3}\left(\mathrm{Sr}_{2} \mathrm{AlTaO}_{6}\right)_{0.7}, \mathrm{SrTiO}_{3}$, and $\mathrm{DyScO}_{3}$, denoted as LAO, LSAT, STO, and DSO, respectively. The plots (Figures 1 and $S 1$ in Supporting Information) contain an intense, sharp peak from the substrate together with the principal reflection of the SMO film, from which its out-ofplane lattice parameter $\left(a_{z}\right)$ can be obtained. Around it, welldefined oscillations are observed in the films below a certain thickness value, which is strain-dependent. The 002 main peak of the film becomes narrower upon increasing thickness, according to Scherrer's equation. Then, above a particular thickness, the coherence fringes vanish and the peak becomes wider and less defined (or even splits into multiple maxima). Such behavior proves the deterioration of the coherent growth of the film through an increased concentration of structural defects, pointing at the beginning of epitaxial strain relaxation. ${ }^{27,28}$ We have used this approach to determine the critical thickness for our SMO films, and the results are shown in Table 1 and Figure 2.

As expected, $t_{c}$ in SMO films decreases with increasing $f$, in agreement with the general trend observed in the epitaxial growth of other materials. ${ }^{26}$ The Matthews-Blakeslee model for misfit dislocations-mediated relaxation ${ }^{29}$ in the case of tensile mismatch $(f>0)$ is often used to calculate the $f\left(t_{c}\right)$ dependence, which can be written as

Table 1. Experimental and Calculated Values of the Critical Thickness $\left(t_{c}\right)$ of Epitaxial $\mathrm{SrMnO}_{3}$ Films on Different Substrates with the Mismatch $(f)$ Indicated $^{a}$

$\begin{array}{crcc}\text { substrate } & \text { mismatch, } f(\%) & \text { experimental } t_{\mathrm{c}}(\mathrm{nm}) & \text { calculated } t_{\mathrm{c}}(\mathrm{nm}) \\ \text { LAO } & -0.39 & 32.5 \pm 2.5 & \text { not applicable } \\ \text { LSAT } & 1.68 & 22.5 \pm 2.5 & 5.3 \\ \text { STO } & 2.63 & 17.5 \pm 2.5 & 2.8 \\ \text { DSO } & 3.78 & 12.5 \pm 2.5 & 1.8\end{array}$

${ }^{a}$ Values calculated according to Matthews-Blakeslee model have been added for comparison (see the text for explanations)

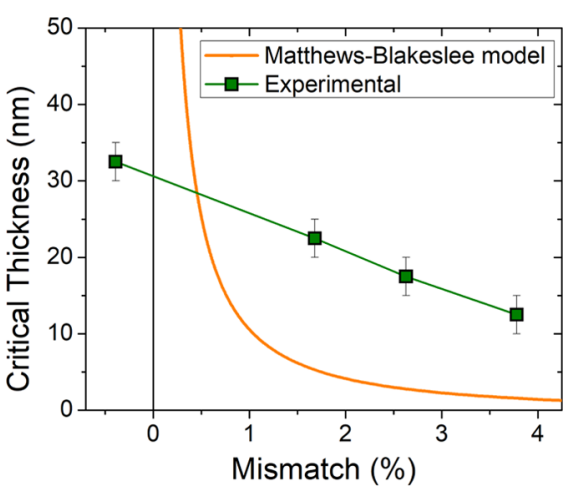

Figure 2. Mismatch dependence of the critical thickness obtained experimentally (solid square symbols) and calculated according to the Matthews-Blakeslee model (orange solid line).

$$
f=\frac{1}{t_{\mathrm{c}}} \cdot \frac{b\left(1-\nu \cdot \cos ^{2} \beta\right)}{8 \pi \cdot(1+\nu) \cdot \cos \lambda} \cdot \ln \left(\frac{\alpha \cdot t_{\mathrm{c}}}{b}\right)
$$

Here, several parameters are related with the dislocation system ( $b$ is the magnitude of Burgers vector, $\lambda$ and $\beta$ are the angles between the dislocation line and the film plane or Burgers vector, respectively), while $\nu$ is Poisson's ratio of the film and $\alpha$ a cutoff parameter. A reasonable estimate for oxide perovskites with cube-on-cube epitaxial growth and a $\{101\}\langle 10-1\rangle$ slip system gives $\lambda=45^{\circ}, \beta=90^{\circ}$, and $\alpha=4 .^{30}$ Taking $b=(\sqrt{2}) \cdot a_{\mathrm{u}}=5.382 \AA, \nu=0.25$ obtained through $\mathrm{ab}$ initio calculations from the elastic constants for isotropic polycrystalline cubic SMO, ${ }^{31}$ the mismatch dependence of $t_{c}$ for the SMO films can then be computed numerically from eq 1 and is shown in Figure 2. The specific computed $t_{c}$ values for the mismatches corresponding to LSAT, STO, and DSO substrates are listed in Table 1 . As observed, the experimental and calculated results greatly differ.

There is wide evidence that Matthews-Blakeslee's model underestimates the critical thickness in many materials as a consequence of a number of simplifications used in its derivation, mainly the neglect of kinetic barriers for the nucleation and propagation of dislocations. ${ }^{32,33}$ Furthermore, the versatile structure of $\mathrm{ABO}_{3}$ perovskite oxides allows a rich variety of additional mechanisms for the relaxation of epitaxial strain, such as twinning, ${ }^{34}$ lack of stoichiometry, ${ }^{17}$ or rotations of the $\mathrm{BO}_{6}$ octahedra, ${ }^{18,35}$ occasionally with complex interactions between them. ${ }^{36}$ In the case of ferroelectrics, another possible way to release the stress is by forming different ferroelectric-ferroelastic domain patterns, whose configuration arises from the epitaxial strain and film thickness. ${ }^{37-39}$ In particular, polar epitaxially strained SMO films on LSAT show both a lateral inhomogeneity in the distribution of oxygen vacancies (which accumulate in the walls separating polar domains) ${ }^{12}$ and concentration gradients along the out-of-plane direction. ${ }^{40}$ This complex scenario makes the application of Matthews-Blakeslee and similar models too simplistic and explains the large discrepancy between the calculated and observed values of $t_{\mathrm{c}}$ in our films.

To further corroborate the experimental $t_{c}$ obtained by the analysis of the $\theta / 2 \theta \mathrm{XRD}$ scans (Figure 1 ), reciprocal space maps (RSM) around the asymmetric $103 \mathrm{XRD}$ reflection of SMO films grown on LSAT and STO substrates (1.7 and $2.6 \%$ epitaxial strain, respectively) were measured and are shown in Figure S2 of the Supporting Information. These plots allow the simultaneous determination of the in-plane, $Q_{x}$, and out-of- 

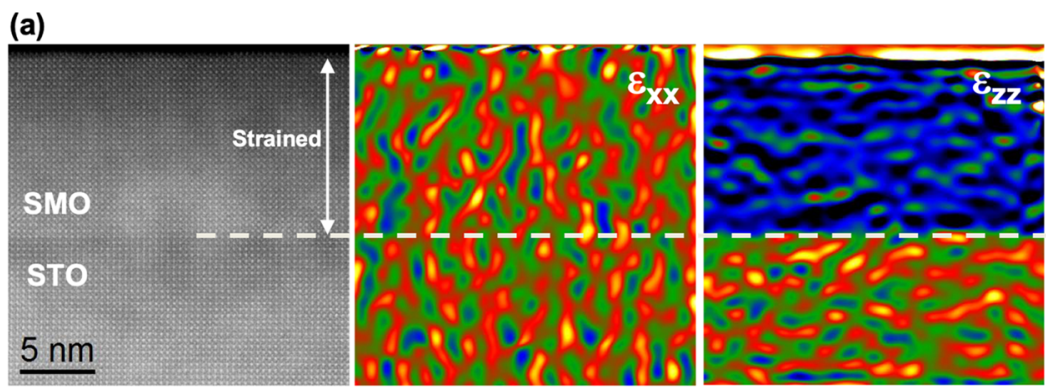

\section{(b)}

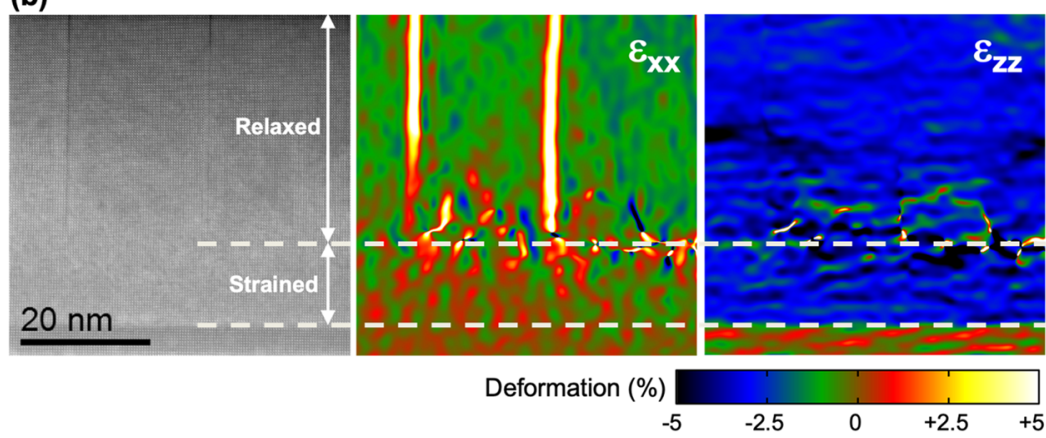

Figure 3. STEM images (left panel) and GPA deformation maps (central panel: in-plane and right panel: out-of-plane) obtained in cross sections of epitaxial SMO films grown on STO, with thicknesses (a) $13 \mathrm{~nm}$ (below $t_{\mathrm{c}}$ ) and (b) $50 \mathrm{~nm}$ (above $t_{\mathrm{c}}$ ).

plane, $Q$, components of the scattering vector $\mathbf{Q}$ of the selected $(h k l)$ XRD reflection, which are directly related to the in-plane and out-of-plane lattice parameters, respectively, as $a_{x}$ $=2 \pi h / Q_{x}$ and $a_{z}=2 \pi l / Q_{z} \cdot{ }^{27}$ As $t_{c}$ corresponds to the thickness above which the in-plane lattice constant of the film starts differing from that of the substrate, it can be inferred from the RSM plots when $Q_{x, \text { film }} \neq Q_{x \text {,substrate }}$. As observed in Figure S2, the $Q_{x}$ component of the 103 reflection of the SMO films starts diverging from that of LSAT at thickness, $t$, equal to $25 \mathrm{~nm}$, and gradually becomes more and more different as $t$ further increases, reflecting the partially relaxed state of the SMO films; instead, for $t \leq 20 \mathrm{~nm}, Q_{x \text {,film }}$ exactly matches $Q_{x, \text { substrate, }}$ evidencing that, in this scenario, SMO has grown fully coherent. Thus, $t_{c}$ should be $22.5 \pm 2.5 \mathrm{~nm}$, in excellent agreement with the range obtained from the symmetrical $\theta / 2 \theta$ scans and presented in Table 1. Similarly, for the SMO films grown on STO, $t_{c}=17.5 \pm 2.5 \mathrm{~nm}$ is inferred from the RSM plots (Figure S2), which is exactly the value obtained from the symmetrical $\theta / 2 \theta$ scans and presented in Table 1 .

Strain Relaxation Mechanisms. To get a thorough understanding of how SMO films relieve the epitaxial stress, the microstructure and strain state of selected STO/SMO films with 13 and $50 \mathrm{~nm}$ thickness (just below and far above $t_{\mathcal{c}}$ respectively) were studied by atomic-resolution scanning transmission electron microscopy (STEM) and geometrical phase analysis (GPA). Representative examples are presented in Figure 3. In the sample with thickness below $t_{c}$ (Figure 3a, left panel), STEM proves that the interfaces are abrupt and SMO has a distorted cubic structure. The deformation values of the films in respect to the substrate shown in the GPA maps are calculated relative to the lattice parameter of the substrate as $^{41}$

$$
\varepsilon_{x x}=\frac{a_{x}-a_{\mathrm{s}}}{a_{\mathrm{s}}}
$$

$$
\varepsilon_{z z}=\frac{a_{z}-a_{\mathrm{s}}}{a_{\mathrm{s}}}
$$

This analysis in the $13 \mathrm{~nm}$ film (Figure 3a, central panel) shows that there is no in-plane deformation $\left(\varepsilon_{x x}=0\right)$ and thus confirms the pseudomorphic growth, i.e., $a_{x}=a_{\mathrm{s}}=3.905 \AA$. The negative out-of-plane deformation of the fully strained SMO film can be observed in the right panel of Figure $3 \mathrm{a}$. The average value $\varepsilon_{z z}=-(4.0 \pm 0.7) \%$ is obtained from eq $2 \mathrm{~b}$ and gives $a_{z} \approx 3.75 \pm 0.03 \AA$, which is lower than $a_{\mathrm{u}}=3.805 \AA$ for unstrained cubic SMO. This behavior, where the in-plane biaxial tensile stress induces a decrease in the out-of-plane parameter of the film relative to the bulk, is commonly observed in epitaxial growth and will be discussed for SMO in detail in the following section. The results of GPA analyses done in similar, coherently strained LSAT/SMO and LAO/ SMO films were published elsewhere. ${ }^{12,14}$

Regarding the 50-nm-thick SMO sample on STO, the STEM image (Figure $3 b$, left panel) shows coherent, almost defect-free growth up to $\approx 15 \mathrm{~nm}$ distance from the substratefilm interface, which is steep and atomically flat. According to the $\varepsilon_{x x}$ GPA deformation map (Figure $3 \mathrm{~b}$, central panel), the film below this thickness presents the same in-plane lattice parameter as the substrate $\left(\varepsilon_{x x} \approx 0\right.$ and thus $a_{x} \approx a_{\mathrm{s}}=3.905$ $\AA$ ). Edge dislocations nucleate at $\approx 15 \mathrm{~nm}$ and evolve upward into stacking faults with missing $\mathrm{MnO}$ planes. Both mechanisms contribute to the release of the accumulated stress. The in-plane $\left(\varepsilon_{x x}\right)$ GPA map above this thickness shows huge positive deformations levels around the dislocation line (reaching values in excess of $+7 \%$ ) and small negative values $\approx-1 \%$ in regions several nanometers distant from those defects and close to the film top surface. This behavior proves that even in the thicker films, the first atomic layers keep the pseudomorphic growth below $t_{\mathrm{c}}$ (determined previously for $\mathrm{STO} / \mathrm{SMO}$ as $17.5 \pm 2.5 \mathrm{~nm}$ from $\theta / 2 \theta$ scans and RSM), and then the strain is released above $t_{\mathrm{c}}$ through the formation of the aforementioned structural defects. The $\varepsilon_{z z}$ map (Figure $3 \mathrm{~b}$, 
Table 2. Lattice Parameters $(a)$ and Strains $(\varepsilon)$ along the In-Plane $(x)$ and Out-of-Plane $(z)$ Direction in Fully Strained SMO Films $^{a}$

\begin{tabular}{|c|c|c|c|c|c|c|}
\hline substrate & $a_{x}(\AA)$ & $a_{z}(\AA)$ & $\varepsilon_{x}=f$ & $\varepsilon_{z}$ & $V\left(\AA^{3}\right)$ & $V_{\mathrm{o}}\left(\AA^{3}\right)$ \\
\hline $\mathrm{LAO}$ & 3.790 & $3.817 \pm 0.002$ & -0.0039 & $0.0032 \pm 0.0005$ & $54.83 \pm 0.03$ & 55.089 \\
\hline LSAT & 3.869 & $3.775 \pm 0.002$ & 0.0168 & $-0.0079 \pm 0.0005$ & $56.51 \pm 0.03$ & 55.089 \\
\hline STO & 3.905 & $3.756 \pm 0.002$ & 0.0263 & $-0.0129 \pm 0.0005$ & $57.28 \pm 0.03$ & 55.089 \\
\hline DSO & 3.949 & $3.743 \pm 0.002$ & 0.0378 & $-0.0163 \pm 0.0005$ & $58.37 \pm 0.03$ & 55.089 \\
\hline
\end{tabular}

${ }^{a}$ Last two columns show the cell volume of the strained unit cell $(V)$ and that of the bulk material $\left(V_{\mathrm{o}}\right)$

right panel) in the relaxed region shows a wide distribution of deformation values between -2.5 and $-4 \%$, corresponding to out-of-plane lattice parameters of the SMO film between $\approx 3.75$ and $\approx 3.81 \AA$, respectively. In excellent agreement, the 002 reflection in the symmetrical $\theta / 2 \theta$ scans of the films on STO with thickness above $30 \mathrm{~nm}$ (Figure $\mathrm{S} 1$ in the Supporting Information) spread in the $2 \theta$ range from 47.8 to $48.5^{\circ}$, corresponding to bulk SMO $\left(a_{\mathrm{u}} \approx 3.81 \AA\right)$ and fully strained films $\left(a_{z} \approx 3.75 \AA\right.$ ) , respectively.

Poisson's Ratio and the Oxygen Content in Fully Strained Films. Below the critical thickness, the films grow coherently strained with the substrate $\left(a_{s}=a_{x}\right)$, which makes the in-plane strain $\left(\varepsilon_{x}\right)$ equal to the mismatch $(f)$

$$
\varepsilon_{x}=\frac{a_{x}-a_{\mathrm{u}}}{a_{\mathrm{u}}}=\frac{a_{\mathrm{s}}-a_{\mathrm{u}}}{a_{\mathrm{u}}}=f
$$

Similarly, the out-of-plane strain $\left(\varepsilon_{z}\right)$ is

$$
\varepsilon_{z}=\frac{a_{z}-a_{\mathrm{u}}}{a_{\mathrm{u}}}
$$

Figure S3 (Supporting Information) shows the $\theta / 2 \theta \mathrm{XRD}$ scans measured in SMO films with thicknesses of $20 \mathrm{~nm}$ on LAO, $20 \mathrm{~nm}$ on LSAT, $15 \mathrm{~nm}$ on STO, and $12.5 \mathrm{~nm}$ on DSO, i.e., below $t_{c}$ in all of the cases. The presence of Von Laue oscillations proves that the films grow with high crystalline quality. The values of $a_{z}$ obtained from these $\theta / 2 \theta$ scans are (see Table 2) $3.817 \pm 0.002 \AA$ on LAO, $3.775 \pm 0.002 \AA$ on LSAT, $3.756 \pm 0.002 \AA$ on STO (in agreement with the previous rough estimate by GPA, $3.75 \pm 0.03 \AA$ ), and $3.743 \pm$ $0.005 \AA$ on DSO. It has to be noted that these values remain constant for every substrate, within the experimental error, in all of the films below $t_{c}$.

As explained before, an in-plane biaxial tensile (compressive) stress in the SMO epitaxial films induces a decrease (increase) in the out-of-plane lattice parameter of the film relative to the bulk. By analogy with the macroscopic materials, a way to formally quantify such "elastic” response is through Poisson's ratio. $^{42}$ In the present case, where the biaxial strain created by the cubic substrate induces a tetragonal distortion on the cubic cell of epitaxial SMO, this magnitude can be calculated as ${ }^{27,32}$

$$
\nu=\frac{1}{1-2 \cdot \frac{\varepsilon_{x}}{\varepsilon_{z}}}
$$

It is very useful to compare the cell volume of the strained unit cell $\left(V=a_{x}^{2} \cdot a_{z}\right)$ with that of the bulk material $\left(V_{\mathrm{o}}=a_{\mathrm{u}}{ }^{3}\right)$. After the previous definitions and using $\varepsilon_{x}=f$, the normalized volume (ratio between both volumes) in the first-order approximation (that is, neglecting the terms in $f^{2}$ and $f^{3}$ ) is

$$
\frac{V}{V_{\mathrm{o}}}=1+2\left(\frac{1-2 \nu}{1-\nu}\right) f
$$

Given that $\nu<0.5$ for all stable materials, ${ }^{42}$ this equation shows that, contrary to the common belief, epitaxial films under in-plane tensile stress $(f>0)$ always have a larger volume than the bulk material. Indeed, the smaller the $\nu$ values, the larger the increase in the volume. Conversely, in-plane compressive strain gives rise to a decrease in the cell volume.

We have used the experimental lattice parameter $a_{z}$ measured in fully coherent films and the nominal values $a_{\mathrm{s}}$ $\left(=a_{x}\right), a_{\mathrm{w}}$ and $f$ to calculate the in-plane and out-of-plane strains and the cell volumes for SMO on all of the substrates. The numerical results are presented in Table 2 and the graphical representation of $V / V_{\mathrm{o}}$ vs. $f$ is shown in Figure 4. Poisson's ratio obtained from the fit through eq 5 is $\nu=0.184$ \pm 0.005 . Note that the excellent fit gives additional proof of the films being fully strained below $t_{\mathrm{c}}$.

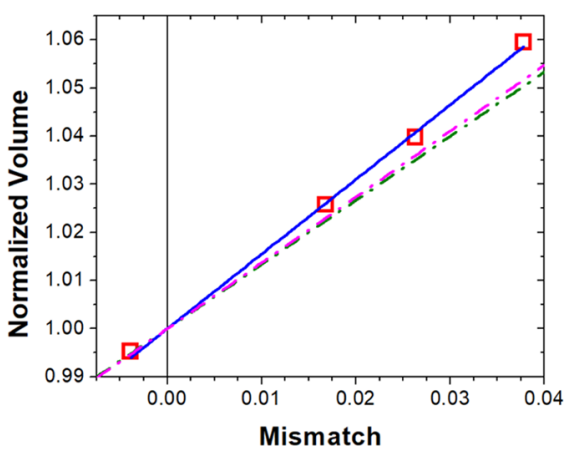

Figure 4. Experimental values (red squares) of the ratio between the measured volume of the strained unit cell $(V)$ in the SMO films and that of the bulk material $\left(V_{\mathrm{o}}\right)$, as a function of the mismatch. The blue straight line is the best fit according to eq 5, from which the experimental Poisson ratio $\nu=0.184 \pm 0.005$ has been calculated. The green and pink lines correspond to $\nu=0.25$ and 0.24 , respectively (see explanations in the text).

Most oxides show typical values of the Poisson ratio in the range $0.20-0.40 .^{42,43}$ In the case of alkaline-earth manganites, Søndenå et al. ${ }^{31}$ obtained for SMO $\nu=0.25$ through ab initio calculations, not far from $\nu=0.23$ measured by XRD in bulk $\mathrm{CaMnO}_{3}{ }^{44}$ A very similar experimental value $\nu=0.24$ was determined in epitaxial SMO through the careful measurement of the lattice parameters in biaxially strained films. ${ }^{25}$ The relative volume vs. mismatch dependence for these two values of $\nu$ for SMO from the literature has been included in Figure 4 for comparison. Quite remarkably, in the experiments by Agrawal et al., ${ }^{25}$ the SMO films were capped with a DSO overlayer to prevent oxygen out-diffusion. This suggests that the tensile-strained SMO films without a capping layer prepared in the present study may suffer significant oxygen loss, in spite of the postdeposition annealing in high partial pressure of $\mathrm{O}_{2}$. Such loss would give rise to an increased concentration of oxygen vacancies and consequently a higher 
$\mathrm{Mn}^{3+} / \mathrm{Mn}^{4+}$ ratio, a larger cell volume due to the higher ionic radius of the $\mathrm{Mn}^{3+}$ with respect to $\mathrm{Mn}^{4+}$, and lower apparent Poisson's ratio $(\nu=0.18)$ than in the stoichiometric films. The large unit cell of our SMO films resulting from the increased amount of $\mathrm{Mn}^{3+}$ cations definitely accommodates substrates with larger lattice parameters more efficiently. This explanation would be in agreement with density functional theory calculations, which show that tensile epitaxial strain in SMO promotes the creation of oxygen vacancies, ${ }^{12,19}$ and thus a decrease in the average valence of $\mathrm{Mn}$, as a mechanism to relieve the epitaxial stress. This may be the reason of the $t_{\mathrm{c}}$ value found in our films grown on DSO substrates being larger (around 25\%) than that found in those films where oxygen out-diffusion is somewhat limited because of the capping layer. $^{25}$

Aiming to confirm these predictions, the oxidation state of the Mn cations of the fully strained SMO films was measured by X-ray photoelectron spectroscopy (XPS). A reliable way to experimentally determine the $\mathrm{Mn}$ valence and thus the oxygen content in manganese-containing oxides is through the split of the Mn 3s peak observed in the X-ray photoelectron spectrum, as described by Galakhov et al. ${ }^{45}$ To ensure that all of the films were coherently strained and that the photoelectrons were emitted by the same volume of material, we carried out these measurements in the same experimental conditions on 5-nmthick films (that is well below $t_{\mathrm{c}}$ ) deposited simultaneously on the four substrates. It has to be noted that XPS provides average information on the uppermost few nanometers, ${ }^{46}$ and thus our results in 5-nm-thick films are representative of the whole film.

Figure 5a shows the X-ray photoelectron spectra measured around the $\mathrm{Mn} 3 \mathrm{~s}$ region. As it has been reviewed in ref 45 , the splitting between the two maxima $(\Delta E)$ correlates with the formal valence of $\mathrm{Mn}$. To carefully determine this splitting, we have fitted the $\mathrm{Mn} 3 \mathrm{~s}$ profile using four Voigt-type symmetric functions. Figure $5 \mathrm{~b}$ shows $\Delta E$ and the formal oxidation state of $\mathrm{Mn}$ as a function of mismatch. The same trend was found using just two components for the fit, but residuals are higher and absolute values are therefore less reliable (details of both fittings can be seen in Figure S4 of the Supporting Information). As it can be seen, an increase of the mismatch is accompanied by a decrease in the average valence of $\mathrm{Mn}$. In other words, the $\mathrm{Mn}^{3+} / \mathrm{Mn}^{4+}$ cation ratio and the number of oxygen vacancies increase with tensile strain. Therefore, these results (i) prove that this anion-point-defect mechanism is very efficient when it comes to accommodating an increasingly larger tensile stress due to the larger average cell size when more and more $\mathrm{Mn}^{3+}$ cations are present and (ii) explains the reduced Poisson's ratio value found in our films compared to that predicted theoretically.

\section{SUMMARY AND CONCLUSIONS}

We have thoroughly analyzed the epitaxial strain relaxation mechanisms in $\mathrm{SrMnO}_{3}$ films. The critical thickness, $t_{c}$, below which pseudomorphic growth is achieved has been systematically determined for different substrates imposing varying degrees of epitaxial strain from -0.4 to $+3.8 \%$. Above $t_{c}$ i.e., when the films become partially relaxed, the formation of dislocations and stacking faults are the main mechanisms for the relief of elastic energy. However, below $t_{\mathcal{c}}$, i.e., for fully tensile-strained films, anion-point-defect relaxation mechanism prevails. Moreover, the presence of these point defects is found to be strain-dependent: the larger the tensile strain, the more

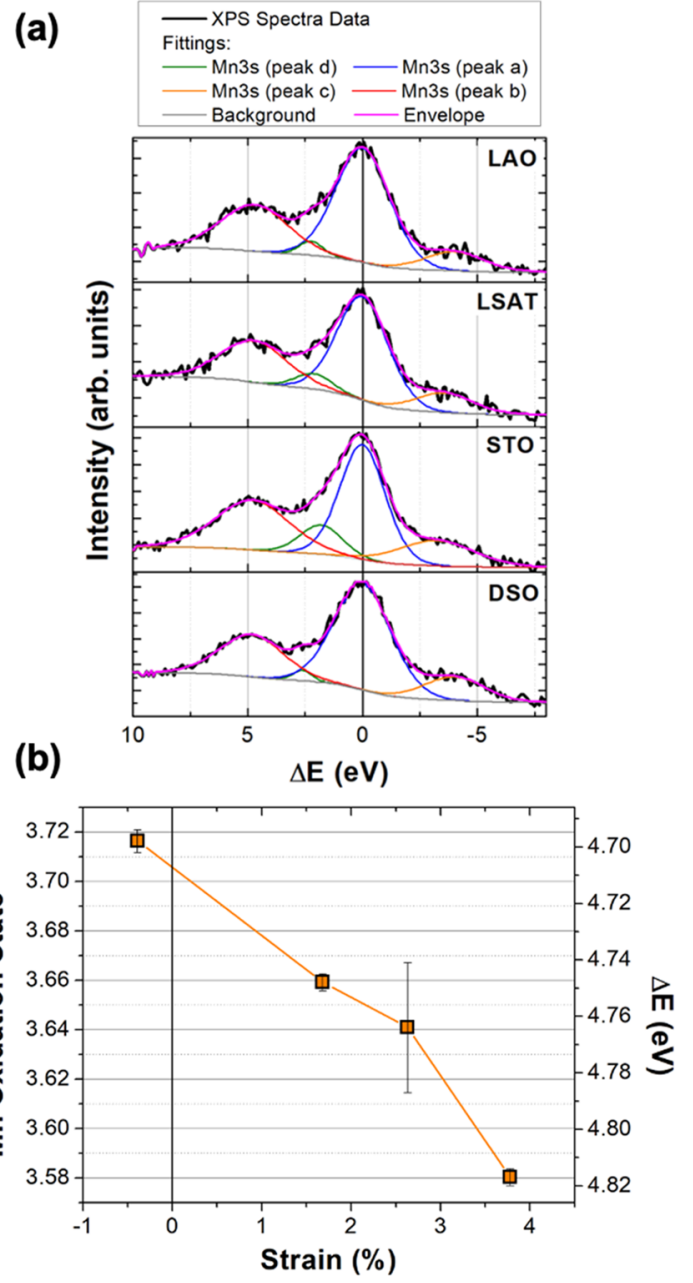

Figure 5. (a) Experimental XPS Mn 3s spectra and the corresponding fittings (see text for explanation) of 5-nm-thick SMO films grown simultaneously on LAO, LSAT, STO, and DSO substrates. The shift of the binding energy $(\Delta E)$ relative to the main peak has been used for the abscissas. (b) Strain dependency of the Mn oxidation state of SMO films (left axis) and of the splitting of the Mn 3s band (right axis). Error bars correspond to the uncertainty resulting from the fitting procedure by slightly varying the background and parameter constraints.

oxygen-deficient the films become. This mechanism arises from the fact that oxygen vacancies induce larger unit cell size in $\mathrm{SrMnO}_{3}$, as they prompt the formation of $\mathrm{Mn}^{3+}$ cations at the expense of relatively smaller $\mathrm{Mn}^{4+}$ cations, which accommodates the increasingly larger lattice-parameter substrates more effectively. This result is in agreement with firstprinciples predictions and indicates that, in practice, the effective in-plane strain could be lower than the nominal mismatch even in coherently grown epitaxial films, hampering the stabilization of some predicted strain-induced ferroic phases. These oxygen vacancies in $\mathrm{SrMnO}_{3}$ films have detrimental implications, as (i) they decrease the effective strain relative to the nominal mismatch and (ii) they increase their electrical conductivity and thus hinder the experimental confirmation of a possible ferroelectric state, as a result of the high leakage currents. Yet, on the other hand, the strain control of oxygen vacancies formation can open an avenue to tune not only the electronic and electrical properties of alkaline-earth 
manganites but also the magnetic ground states resulting from the different $\mathrm{Mn}^{3+} / \mathrm{Mn}^{4+}$ magnetic cation ratios.

\section{EXPERIMENTAL SECTION}

Thin-Film Growth. Epitaxial SMO films of different thickness $(t)$ between 5 and $100 \mathrm{~nm}$ were grown by pulsed laser deposition (PLD) on commercially available substrates with a nearly cubic perovskite structure: $\mathrm{LaAlO}_{3}$, (LaA$\left.1 \mathrm{O}_{3}\right)_{0.3}\left(\mathrm{Sr}_{2} \mathrm{AlTaO}_{6}\right)_{0.7}, \mathrm{SrTiO}_{3}$, and $\mathrm{DyScO}_{3}$, denoted as LAO, LSAT, STO, and DSO, respectively. They all had (001) orientation in the pseudocubic-axes notation that will be used hereinafter and lattice parameters $3.790 \AA$ (LAO), $3.869 \AA$ (LSAT), $3.905 \AA$ (STO), and $3.949 \AA$ (DSO). All of the films with the same thickness were deposited simultaneously on LAO, LSAT, STO, and DSO substrates to ensure that they have the same cation stoichiometry. The lattice mismatch between the film and each of the substrates can be calculated as

$$
f=\frac{a_{\mathrm{s}}-a_{\mathrm{u}}}{a_{\mathrm{u}}}
$$

where $a_{s}$ is the pseudocubic lattice parameter of the substrate and $a_{\mathrm{u}}$ is the unstrained lattice constant of the film material. Taking $a_{\mathrm{u}}=3.805 \AA$ for bulk cubic $\mathrm{SMO}^{47}$ gives $f$ values $-0.39 \%$ (LAO), $+1.68 \%$ for LSAT, $+2.63 \%$ for STO, and $+3.78 \%$ for DSO. The positive values indicate that the substrate imposes biaxial tensile stress on the plane of the film. LAO was chosen because it presents a very small mismatch relative to SMO so these films can be considered nearly strain-free although they are slightly compressed inplane.

PLD was carried out using a $\mathrm{KrF}$ excimer laser (248 nm wavelength) with $0.8 \mathrm{~J} / \mathrm{cm}^{2}$ fluence on the target, 0.1 Torr dynamic oxygen pressure, and $850{ }^{\circ} \mathrm{C}$ substrate temperature, as explained in detail elsewhere. ${ }^{12}$ To keep the oxygen content of the films as high as possible, the as-grown samples were cooled to $750{ }^{\circ} \mathrm{C}$ in the deposition pressure and then annealed in 700 Torr oxygen for $30 \mathrm{~min}$, followed by slow cooling to room temperature in the same atmosphere.

Structural Characterization. X-ray diffraction (XRD) measurements, including symmetric $\theta / 2 \theta$ scans and the reciprocal space maps (RSM), were carried out in a Bruker D8 Advance high-resolution diffractometer with parallel-beam optics and $\mathrm{Cu} \mathrm{K} \alpha_{1}$ radiation (wavelength $1.54056 \AA$ ) to determine the crystal structure and quality of the films. The thickness of the films was measured by X-ray reflectivity (XRR) in the same experimental setup.

The local microstructure was observed by scanning transmission electron microscopy (STEM) on a probe-corrected FEI Titan 60-300 microscope equipped with a high-brightness field-emission gun (X-FEG) and a CEOS aberration corrector for the condenser system. This microscope was operated at $300 \mathrm{kV}$. Atomic-resolution $\mathrm{Z}$ contrast images were obtained by high-angle annular dark-field (HAADF) imaging in STEM with a convergence angle of $25 \mathrm{mrad}$ and a probe size below 1 $\AA$. The strain state of the SMO films with respect to the substrate along the in-plane $\left(\varepsilon_{x x}\right)$ and out-of-plane $\left(\varepsilon_{z z}\right)$ directions was determined through geometrical phase analysis (GPA) of the HAADF images. ${ }^{41}$

Mn Oxidation State. The Mn valence was determined by XPS in a Kratos Axis SUPRA spectrometer employing a monochromatic $\mathrm{Al} \mathrm{K} \alpha(1486.6 \mathrm{eV}) \mathrm{X}$-ray source. The lateral size of the region explored was $0.7 \times 0.3 \mathrm{~mm}^{2}$. The spectra were analyzed using Casa software, including background subtraction of a Shirley baseline. The oxidation state of manganese was calculated from its direct relationship with the splitting of the Mn 3s peaks, as established by Galakhov et al. ${ }^{45}$

\section{ASSOCIATED CONTENT}

\section{Supporting Information}

The Supporting Information is available free of charge at https://pubs.acs.org/doi/10.1021/acsomega.1c00953.

Additional XRD results $(\theta / 2 \theta$ scans and reciprocal space maps) and XPS measurements and fittings (PDF)

\section{AUTHOR INFORMATION}

\section{Corresponding Authors}

Eric Langenberg - Instituto de Nanociencia y Materiales de Aragón (INMA), CSIC-Universidad de Zaragoza, 50009 Zaragoza, Spain; Departamento de Física de la Materia Condensada, Universidad de Zaragoza, 50009 Zaragoza, Spain; (이이.org/0000-0002-6944-4713;

Email: eric.langenberg.perez@gmail.com

José A. Pardo - Instituto de Nanociencia y Materiales de Aragón (INMA), CSIC-Universidad de Zaragoza, 50009 Zaragoza, Spain; Laboratorio de Microscopías Avanzadas, Universidad de Zaragoza, 50018 Zaragoza, Spain; Departamento de Ciencia y Tecnología de Materiales y Fluidos, Universidad de Zaragoza, 50018 Zaragoza, Spain; Email: jpardo@unizar.es

\section{Authors}

Laura Maurel - Instituto de Nanociencia y Materiales de Aragón (INMA), CSIC-Universidad de Zaragoza, 50009 Zaragoza, Spain

Guillermo Antorrena - Laboratorio de Microscopías Avanzadas, Universidad de Zaragoza, 50018 Zaragoza, Spain

Pedro A. Algarabel - Instituto de Nanociencia y Materiales de Aragón (INMA), CSIC-Universidad de Zaragoza, 50009 Zaragoza, Spain; Departamento de Física de la Materia Condensada, Universidad de Zaragoza, 50009 Zaragoza, Spain

César Magén - Instituto de Nanociencia y Materiales de Aragón (INMA), CSIC-Universidad de Zaragoza, 50009 Zaragoza, Spain; Departamento de Física de la Materia Condensada, Universidad de Zaragoza, 50009 Zaragoza, Spain; Laboratorio de Microscopías Avanzadas, Universidad de Zaragoza, 50018 Zaragoza, Spain; ㅇorcid.org/00000002-6761-6171

Complete contact information is available at: https://pubs.acs.org/10.1021/acsomega.1c00953

\section{Author Contributions}

E.L. and L.M. contributed equally. This manuscript was written through the contributions of all authors. All authors have given approval to the final version of the manuscript.

Notes

The authors declare no competing financial interest.

\section{ACKNOWLEDGMENTS}

This work was financially supported by the Spanish Ministry of Science through project MAT2017-82970-C2-1-R and MAT2017-82970-C2-2-R and the Aragón Regional Government through projects E13_20R and E28_20R (Construyendo 
Europa desde Aragón). This project received funding from the European Union's Horizon 2020 research and innovation program under grant agreement no. 823717-ESTEEM3. The diffraction, microscopy, and XPS experiments were conducted in the Laboratorio de Microscopías Avanzadas (LMA), Universidad de Zaragoza.

\section{REFERENCES}

(1) Matsukura, F.; Tokura, Y.; Ohno, H. Control of Magnetism by Electric Fields. Nat. Nanotechnol. 2015, 10, 209-220.

(2) Song, C.; Cui, B.; Li, F.; Zhou, X.; Pan, F. Recent Progress in Voltage Control of Magnetism: Materials, Mechanisms, and Performance. Prog. Mater. Sci. 2017, 87, 33-82.

(3) Bhattacharjee, S.; Bousquet, E.; Ghosez, P. Engineering Multiferroism in $\mathrm{CaMnO}_{3}$. Phys. Rev. Lett. 2009, 102, No. 117602.

(4) Lee, J. H.; Rabe, K. M. Epitaxial-Strain-Induced Multiferroicity in $\mathrm{SrMnO}_{3}$ from First Principles. Phys. Rev. Lett. 2010, 104, No. 207204.

(5) Rondinelli, J. M.; Eidelson, A. S.; Spaldin, N. A. Non- $d^{0}$ MnDriven Ferroelectricity in Antiferromagnetic $\mathrm{BaMnO}_{3}$. Phys. Rev. $B$ 2009, 79, No. 205119.

(6) Günter, T.; Bousquet, E.; David, A.; Boullay, P.; Ghosez, P.; Prellier, W.; Fiebig, M. Incipient Ferroelectricity in $2.3 \%$ TensileStrained $\mathrm{CaMnO}_{3}$ Films. Phys. Rev. B 2012, 85, No. 214120.

(7) Sakai, H.; Fujioka, J.; Fukuda, T.; Okuyama, D.; Hashizume, D.; Kagawa, F.; Nakao, H.; Murakami, Y.; Arima, T.; Baron, A. Q. R.; Taguchi, Y.; Tokura, Y. Displacement-Type Ferroelectricity with offCenter Magnetic Ions in Perovskite $\mathrm{Sr}_{1-\mathrm{x}} \mathrm{Ba}_{\mathrm{x}} \mathrm{MnO}_{3}$. Phys. Rev. Lett. 2011, 107, No. 137601.

(8) Pratt, D. K.; Lynn, J. W.; Mais, J.; Chmaissem, O.; Brown, D. E.; Kolesnik, S.; Dabrowski, B. Neutron Scattering Studies of the Ferroelectric Distortion and Spin Dynamics in the Type-1 Multiferroic Perovskite $\mathrm{Sr}_{0.56} \mathrm{Ba}_{0.44} \mathrm{MnO}_{3}$. Phys. Rev. $B$ 2014, 90, No. 140401.

(9) Goian, V.; Langenberg, E.; Marcano, N.; Bovtun, V.; Maurel, L.; Kempa, M.; Prokscha, T.; Kroupa, J.; Algarabel, P. A.; Pardo, J. A.; Kamba, S. Spin-Phonon Coupling in Epitaxial $\mathrm{Sr}_{0.6} \mathrm{Ba}_{0.4} \mathrm{MnO}_{3}$ Thin Films. Phys. Rev. B 2017, 95, No. 075126.

(10) Langenberg, E.; Maurel, L.; Marcano, N.; Guzmán, R.; Štrichovanec, P.; Prokscha, T.; Magén, C.; Algarabel, P. A.; Pardo, J. A. Controlling the Electrical and Magnetoelectric Properties of Epitaxially Strained $\mathrm{Sr}_{1-\mathrm{x}} \mathrm{Ba}_{\mathrm{x}} \mathrm{MnO}_{3}$ Thin Films. Adv. Mater. Interfaces 2017, 4, No. 1601040.

(11) Langenberg, E.; Guzmán, R.; Maurel, L.; Martínez De Baños, L.; Morellón, L.; Ibarra, M. R.; Herrero-Martín, J.; Blasco, J.; Magén, C.; Algarabel, P. A.; Pardo, J. A. Epitaxial Stabilization of the Perovskite Phase in $\left(\mathrm{Sr}_{1-\mathrm{x}} \mathrm{Ba}_{\mathrm{x}}\right) \mathrm{MnO}_{3}$ Thin Films. ACS Appl. Mater. Interfaces 2015, 7, 23967-23977.

(12) Becher, C.; Maurel, L.; Aschauer, U.; Lilienblum, M.; Magén, C.; Meier, D.; Langenberg, E.; Trassin, M.; Blasco, J.; Krug, I. P.; Algarabel, P. A.; Spaldin, N. A.; Pardo, J. A.; Fiebig, M. Strain-Induced Coupling of Electrical Polarization and Structural Defects in $\mathrm{SrMnO}_{3}$ Films. Nat. Nanotechnol. 2015, 10, 661-666.

(13) Guo, J. W.; Wang, P. S.; Yuan, Y.; He, Q.; Lu, J. L.; Chen, T. Z.; Yang, S. Z.; Wang, Y. J.; Erni, R.; Rossell, M. D.; Gopalan, V.; Xiang, H. J.; Tokura, Y.; Yu, P. Strain-Induced Ferroelectricity and SpinLattice Coupling in $\mathrm{SrMnO}_{3}$ Thin Films. Phys. Rev. B 2018, 97, No. 235135.

(14) Maurel, L.; Marcano, N.; Prokscha, T.; Langenberg, E.; Blasco, J.; Guzmán, R.; Suter, A.; Magén, C.; Morellón, L.; Ibarra, M. R.; Pardo, J. A.; Algarabel, P. A. Nature of Antiferromagnetic Order in Epitaxially Strained Multiferroic $\mathrm{SrMnO}_{3}$ Thin Films. Phys. Rev. B 2015, 92, No. 024419.

(15) Edström, A.; Ederer, C. First-Principles-Based Strain and Temperature-Dependent Ferroic Phase Diagram of $\mathrm{SrMnO}_{3}$. Phys. Rev. Mater. 2018, 2, No. 104409.
(16) Schlom, D. G.; Chen, L. Q.; Fennie, C. J.; Gopalan, V.; Muller, D. A.; Pan, X.; Ramesh, R.; Uecker, R. Elastic Strain Engineering of Ferroic Oxides. MRS Bull. 2014, 39, 118-130.

(17) Herklotz, A.; Lee, D.; Guo, E. J.; Meyer, T. L.; Petrie, J. R.; Lee, H. N. Strain Coupling of Oxygen Non-Stoichiometry in Perovskite Thin Films. J. Phys.: Condens. Matter 2017, 29, No. 493001.

(18) Aschauer, U.; Pfenninger, R.; Selbach, S. M.; Grande, T.; Spaldin, N. A. Strain-Controlled Oxygen Vacancy Formation and Ordering in $\mathrm{CaMnO}_{3}$. Phys. Rev. B 2013, 88, No. 054111.

(19) Marthinsen, A.; Faber, C.; Aschauer, U.; Spaldin, N. A.; Selbach, S. M. Coupling and Competition between Ferroelectricity, Magnetism, Strain, and Oxygen Vacancies in $\mathrm{AMnO}_{3}$ Perovskites. MRS Commun. 2016, 6, 182-191.

(20) Chandrasena, R. U.; Yang, W.; Lei, Q.; Delgado-Jaime, M. U.; Wijesekara, K. D.; Golalikhani, M.; Davidson, B. A.; Arenholz, E.; Kobayashi, K.; Kobata, M.; de Groot, F. M. F.; Aschauer, U.; Spaldin, N. A.; Xi, X.; Gray, A. X. Strain-Engineered Oxygen Vacancies in $\mathrm{CaMnO}_{3}$ Thin Films. Nano Lett. 2017, 17, 794-799.

(21) Shannon, R. D. Revised Effective Ionic Radii and Systematic Studies of Interatomic Distances in Halides and Chalcogenides. Acta Crystallogr., Sect. A 1976, 32, 751-767.

(22) Edström, A.; Ederer, C. Prediction of a Giant Magnetoelectric Cross-Caloric Effect around a Tetracritical Point in Multiferroic $\mathrm{SrMnO}_{3}$. Phys. Rev. Lett. 2020, 124, No. 167201.

(23) Wang, F.; Zhang, Y. Q.; Bai, Y.; Liu, W.; Zhang, H. R.; Wang, W. Y.; Li, S. K.; Ma, S.; Zhao, X. G.; Sun, J. R.; Wang, Z. H.; Wang, Z. J.; Zhang, Z. D. Oxygen Vacancy Formation, Crystal Structures, and Magnetic Properties of Three $\mathrm{SrMnO}_{3-\delta}$ Films. Appl. Phys. Lett. 2016, 109, No. 052403.

(24) Bai, J.; Yang, J.; Dong, W.; Zhang, Y.; Bai, W.; Tang, X. Structural and Magnetic Properties of Perovskite $\mathrm{SrMnO}_{3}$ Thin Films Grown by Molecular Beam Epitaxy. Thin Solid Films 2017, 644, 5764.

(25) Agrawal, P.; Guo, J.; Yu, P.; Hébert, C.; Passerone, D.; Erni, R.; Rossell, M. D. Strain-Driven Oxygen Deficiency in Multiferroic $\mathrm{SrMnO}_{3}$ Thin Films. Phys. Rev. B 2016, 94, No. 104101.

(26) Ohring, M. Materials Science of Thin Films, 2nd ed.; Academic Press: San Diego, USA, 2001.

(27) Birkholz, M. Thin Film Analysis by X-Ray Scattering; Wiley: Weinheim, Germany, 2006.

(28) Kaganer, V.; Köhler, R.; Schmidbauer, M.; Opitz, R.; Jenichen, B. X-Ray Diffraction Peaks Due to Misfit Dislocations in Heteroepitaxial Structures. Phys. Rev. B 1997, 55, No. 1793.

(29) Matthews, J. W.; Blakeslee, A. E. Defects in Epitaxial Multilayers. J. Cryst. Growth 1974, 27, 118-125.

(30) Speck, J. S.; Pompe, W. Domain Configurations Due to Multiple Misfit Relaxation Mechanisms in Epitaxial Ferroelectric Thin Films. I. Theory. J. Appl. Phys. 1994, 76, 466-476.

(31) Søndenå, R. Connectivity of Polyhedra in $\mathrm{AMnO}_{3}(\mathrm{~A}=\mathrm{Ca}, \mathrm{Sr}$, $\mathrm{Ba})$ and $\mathrm{AB}_{2}(\mathrm{~A}=\mathrm{Si}, \mathrm{Ge} ; \mathrm{B}=\mathrm{S}, \mathrm{Se})$. University of Oslo, $2007 \mathrm{https}$ // folk.universitetetioslo.no/ravi/allpapers/106-elastic_SrMnO3.pdf.

(32) Narayan, J. Recent Progress in Thin Film Epitaxy across the Misfit Scale (2011 Acta Gold Medal Paper). Acta Mater. 2013, 61, $2703-2724$.

(33) Wang, T.; Ganguly, K.; Marshall, P.; Xu, P.; Jalan, B. Critical Thickness and Strain Relaxation in Molecular Beam Epitaxy-Grown $\mathrm{SrTiO}_{3}$ Films. Appl. Phys. Lett. 2013, 103, No. 212904.

(34) Pailloux, F.; Lyonnet, R.; Maurice, J. L.; Contour, J. P. Twinning and Lattice Distortions in the Epitaxy of $\mathrm{La}_{0.67} \mathrm{Sr}_{0.33} \mathrm{MnO}_{3}$ Thin Films on (lo 0 1) SrTiO ${ }_{3}$. Appl. Surf. Sci. 2001, 177, 263-267.

(35) May, S. J.; Kim, J.-W.; Rondinelli, J. M.; Karapetrova, E.; Spaldin, N. A.; Bhattacharya, A.; Ryan, P. J. Quantifying Octahedral Rotations in Strained Perovskite Oxide Films. Phys. Rev. B 2010, 82, No. 014110.

(36) Sandiumenge, F.; Santiso, J.; Balcells, L.; Konstantinovic, Z.; Roqueta, J.; Pomar, A.; Espinós, J. P.; Martínez, B. Competing Misfit Relaxation Mechanisms in Epitaxial Correlated Oxides. Phys. Rev. Lett. 2013, 110, No. 107206. 
(37) Pompe, W.; Gong, X.; Suo, Z.; Speck, J. S. Elastic Energy Release Due to Domain Formation in the Strained Epitaxy of Ferroelectric and Ferroelastic Films. J. Appl. Phys. 1993, 74, 60126019.

(38) Kwak, B. S.; Erbil, A.; Budai, J. D.; Chisholm, M. F.; Boatner, L. A.; Wilkens, B. J. Domain Formation and Strain Relaxation in Epitaxial Ferroelectric Heterostructures. Phys. Rev. B 1994, 49, No. 14865.

(39) Langenberg, E.; Paik, H.; Smith, E. H.; Nair, H. P.; Hanke, I.; Ganschow, S.; Catalan, G.; Domingo, N.; Schlom, D. G. StrainEngineered Ferroelastic Structures in $\mathrm{PbTiO}_{3}$ Films and Their Control by Electric Fields. ACS Appl. Mater. Interfaces 2020, 12, 20691-20703.

(40) Guzmán, R.; Maurel, L.; Langenberg, E.; Lupini, A. R.; Algarabel, P. A.; Pardo, J. A.; Magén, C. Polar-Graded Multiferroic $\mathrm{SrMnO}_{3}$ Thin Films. Nano Lett. 2016, 16, 2221-2227.

(41) Hÿtch, M. J.; Snoeck, E.; Kilaas, R. Quantitative Measurement of Displacement and Strain Fields from HREM Micrographs. Ultramicroscopy 1998, 74, 131-146.

(42) Greaves, G. N.; Greer, A. L.; Lakes, R. S.; Rouxel, T. Poisson's Ratio and Modern Materials. Nat. Mater. 2011, 10, 823-837.

(43) Rouxel, T. Elastic Properties and Short-to Medium-Range Order in Glasses. J. Am. Ceram. Soc. 2007, 90, 3019-3039.

(44) Buch, J. J. U.; Lalitha, G.; Pathak, T. K.; Vasoya, N. H.; Lakhani, V. K.; Reddy, P. V.; Kumar, R.; Modi, K. B. Structural and Elastic Properties of Ca-Substituted $\mathrm{LaMnO}_{3}$ at 300 K. J. Phys. D: Appl. Phys. 2008, 41, No. 025406.

(45) Galakhov, V. R.; Falub, M. C.; Kuepper, K.; Neumann, M. XRay Spectroscopy of Lanthanum Manganites: Nature of Doping Holes, Correlation Effects, and Orbital Ordering. J. Struct. Chem. 2008, 49, 54-58.

(46) Briggs, D.; Grant, J. T. Surface Analysis by Auger and X-ray Photoelectron Spectroscopy; IM Publications: Chichester, UK, 2003.

(47) Chmaissem, O.; Dabrowski, B.; Kolesnik, S.; Mais, J.; Brown, D. E.; Kruk, R.; Prior, P.; Pyles, B.; Jorgensen, J. D. Relationship between Structural Parameters and the Néel Temperature in $\mathrm{Sr}_{1-\mathrm{x}} \mathrm{Ca}_{\mathrm{x}} \mathrm{MnO}_{3}(0 \leq \mathrm{x} \leq 1)$ and $\mathrm{Sr}_{1-\mathrm{y}} \mathrm{Ba}_{\mathrm{y}} \mathrm{MnO} 3(\mathrm{y} \leq 0.2)$. Phys. Rev. B 2001, 64, No. 134412. 